\title{
USO DE ALIMENTOS Y COMPETICION ENTRE EL MONO NOCTURNO Aotus vociferans Y OTROS MAMIFEROS, LORETO, PERU
}
P. Puertas
R. A quino*
F. Encarnación ${ }^{*}$

\section{RESUMEN}

Estudios realizados en las cuencas de los ríos $\mathrm{N}$ apo y N anay entre 1986 y 1987 han permitido el registro de plantas cuyos frutos fueron usados por A otus vociferans y otros mamíferos nocturnos. Ellos consumieron mayormente frutos $(83 \%)$ y en menor proporción tlores néctar $(17 \%)$. L os frutos de mayor consumo correspondieron a Ficus sp. (14\%) e higa marginata (10\%). Entre las flores y néctar destaca por su consumo Eschweilera sp. (10\%).

El reporte también incluye descripciones sobre competencia intraespecífica y con otros mamíferos de hábitos similares.

Palabras claves: A otus vociferans, competidores, estudio de campo, plantas alimenticias.

\section{ABST RACT}

During the studies carried out between 1986 and 1987 in the basin of the Napo and Nanay rivers, we have registered plants whose fruits are used by Aotus vociferans and other nocturnal mamals. A otus ate $83 \%$ fruits and $17 \%$ flowers and nectar. Fruits of Ficus sp. (14\%) and Inga marginata (10\%) Was most frequently eaten. A mong flowers and nectar, only Schweilera sp.0\%) was eaten.

Palabras claves: $\quad$ A otus vociferans, competitors, feeding, plants, field study

\section{INTRODUCCION}

De acuerdo a las referencias bibliográficas Aotus además de consumir frutos también se alimentan de hojas tiernas, brotes, néctar, mamíferos pequeños, huevos de aves, capullos e insectos (Hladik et al. 1971) en Wright 1985,

\footnotetext{
Instituto V eterinario de Investigaciones Tropicales y de Altura (IV ITA), A partado postal 575. Iquitos-Perú.
} 
Hernández Camacho y Cooper 1976; Wrigh 1978, 1981, 1985; García y Braza 1987). Aotus también tiene como competidores por los alimentos a otros animales de hábitos similares. Al respecto M oynihan (1964), Rathbun y Gaché (1977) y W right $(1978,1985)$ dan algunos alcances que consideramos aún insuficientes. Esto motivó a los autores a continuar los estudios, cuyos resultados presentamos en este documento como un aporte al conocimiento de la ecología de estos primates.

\section{AREA DE ESTUDIO}

Este estudio se realizó en áreas correspondientes a la desembocadura del

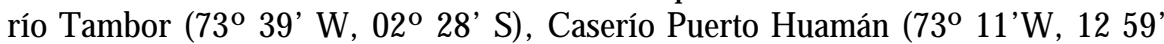

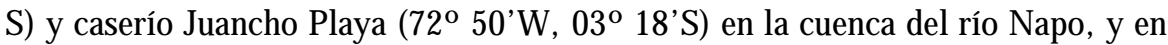
el caserío M ishana (730 28' W, 030 52'S) en la cuenca del río N anay (Fig. 1).

En la cuenca del río $\mathrm{Napo}$, el terreno presenta un relieve ligeramente ondulado, cuyo bosque en las partes bajas corresponde al típico de tahuampa, que en época de creciente es inundado por masas de agua blanca (Encarnación 1985). En el bosque diferenciamos un estrato inferior entre 7 y $15 \mathrm{~m}$ de al to, compuesto por arbustos con troncos erguidos y delgados; un estrato medio entre 16 a $20 \mathrm{~m}$ compuesto por árboles con tronco y copa de mayor diámetro, y un estrato superior entre 21 y $30 \mathrm{~m}$, con presencia de árboles maduros, semierguidos y senescentes, que soportan lianas y epífitos leñosos. También existen árboles emergentes dispersos que sobrepasan los $35 \mathrm{~m}$ de alto. En general, el bosque presenta aspecto degradado cuya presión de caza es al ta que incluye A otus. Entre la vegetación destacan por su predominancia Brosimum guianensis 'huayracaspi , Brosium sp. "tamamuri" Calycophyllum spruceanum "capirona", Clarisia sp. 'capinuri, Diplothropis sp. "bushilla', Eschweilera sp. "machimango", Ficus sp. renaco", Macrolobium ocaciaefulium "pashaco negro", Pithecellobium sp. pashaco", y Scheelea sp. "shapaja".

En la cuenca del río Nanay el terreno cuyo relieve casi plano es inundado estacionalmente por masas de agua negra, los estratos del bosque presentan cierta similitud con los del Napo; pero existe mayor degradación debido a la intensa tala selectiva de árboles de valor comercial. La presión de caza es igualmente alta e incluye Aotus. La vegetación esta representada principalmente por Aspidosperma sp. "remocaspi ", Diplothropis sp., Eschweilera sp., M. acaciefolium, Mauritia flexuosa "aguaje", Pithecellobium sp., P therocarpus amazonicus "maría buena", Sapium aereun "shiringarana", y Vochysia sp. "quillosisa'. 


\section{METODOS}

Este estudio fue ejecutado en el río $\mathrm{Napo}$, desde enero a marzo y noviembre a diciembre de 1986 y en el río N anay, desde abril a julio de 1987.

Las observaciones fueron hechas entre las 03 a 06 horas y entre las 18 a 22 horas. Para el registro de datos fueron necesarios seguimientos sigilosos a los animales; a pie durante el estiaje por senderos previamente aperturados, y en canoa durante la creciente de los ríos.

LoS grupos familiares fueron ubicados por audición de sus vocalizaciones y ruidos característicos producidos durante las actividades alimenticias y de locomoción. Localizado un grupo, el paso siguiente consistió en el seguimiento sigiloso y a una distancia prudente que varió entre 10 a $15 \mathrm{~m}$. El seguimiento nocturno nos permitió reconocer la planta alimenticia por la caída de restos de frutos y flores que fueron verificadas por las huellas dentales dejadas en las cáscaras de los frutos así como en los pedúnculos, sépalos y pétalos de las flores. Luego procedimos a marcar con cinta plástica el fuste de cada árbol, para facilitar su reconocimiento en el día y colectar el material biológico para la identificación taxonómica de la planta. Oportunamente también descubrimos otros mamíferos nocturnos, que compiten con el alimento. Para la interpretación de nuestros resultados hemos considerado la ocurrencia de encuentros entre A otus y los demás mamíferos consumiendo tal o cual recurso alimenticio.

\section{RESULTADOS Y DISCUSION}

En 320 horas de seguimiento nocturno, Aotus vociferans fue observado comiendo frutos, flores y néctares pertenecientes a 16 especies (Tabla 1). Correspondiendo las utilizadas a las familias M oraceae (31 \%) y L egurninosae (21 \%), coincidente con lo reportado por Wright (1985) para A. nigriceps (trivirgatus) en Cocha Cashú, Perú, quién además menciona a las familias Annonaceae, bombacaceae, y Sapindaceae. De las especies registradas, el $83 \%$ correpondieron a flores y néctar. Los frutos de mayor consumo pertenecieron a Ficus sp. (14\%) e higa marginata (10\%), y entre las flores y néctar a EschweiL era sp. (10\%).

Los frutos generalmente fueron consumidos en su estado maduro, aunque también consumieron frutos inrnaduros. La parte más aprovechada por el animal fue el mesocarpio, descartándose el pericarpio y las semillas, con excepción de los frutos pequeños como Coccoloba sp. y Pourourna niinor, que se caracterizan por presentar el pericarpio blando, delgado y las semillas diminutas. Referente al sabor, la mayoría de los frutos consumidos fueron dulces y agradables, y solamente en muy pocas ocasiones, cuando inmaduros, como Ficus sp., fueron insípidos. De las flores, las partes usadas correspondieron al pedúnculo y la porción terminal de la corola. 
Nuestros registros corroboran a los reportados para $A$. lemurinus (Hernández Camacho y Cooper 1976), A. nigriceps (W right 1978, 1981, 1985; Terborgh y Janson 1983, Terborgh 1985), A. azarae (Rathbun y Gaché 1980, Wright 1985, García y Braza 1987) y A. nancyrnae (Hershkovitz 1983), que además de los mencionados, también consumen hojas tiernas, brotes, mamíferos pequeños, huevos de aves, capullos e insectos.

\section{Competición intra-específica}

Casos de competición intra-específica, denominada también competicion intergrupal-agresiva (Janson y Schaik 1988), fueron observados en dos oportunidades en $\mathrm{M}$ ishana, río $\mathrm{N}$ anay. A mbas competiciones ocurrieron por el alimento. La primera ocurrió a las 04:05 h cuando dos grupos integrados por tres individuos cada uno, compartían un mismo árbol de Protium sp.; inicialmente ambos estaban sepa rados por más de $15 \mathrm{~m}$., repentinamente, un adulto de cada grupo, posiblemente los machos, se aproximaron emitiendo vocalizaciones agresivas o de hostigamiento. La confrontación vocal duró 4 minutos, hasta que uno de los grupos opté por la retirada, mientras que el otro continué alimentándose en el árbol por espacio de 14 minutos. El segundo caso ocurrió a las 03:47 h entre dos grupos de tres y cuatro individuos, respectivamente, en las inmediaciones de un bosque secundario con predominancia de árboles de Bellucia aximanthera. En un árbol cuya ramas esparcidas y cargadas de frutos maduros se encontraba alimentándose el grupo compuesto por tres individuos, minutos después, hizo su aparición el grupo de cuatro Individuos, ocupando otro árbol de Bellucia y a una distancia de $10 \mathrm{~m}$ del primero, iniciándose luego la confrontación vocal por espacio de tres minutos, pero no hubo. Agresiones físicas. Posteriormente y casi en forma simultánea los grupos abandonaron los respectivos árboles, para luego desplazarse en distintas direcciones.

De las observaciones realizadas, asumimos que estas competiciones ocurren cuando hay invasión o superposición parcial de sus territorios y coincidente con la temporada de escasez de frutos. Casos de competición por el recurso alimenticio tambien fueron observados por otros investigadores. Moynihan (1964) en Panamá, reporta del hostigamiento entre dos grupos familiares de A. lemurinus (trivirgatus). Por otro lado, Rathbun y Gaché (1977) en A rgentina, inicialmente observaron alimentándose en un mismo árbol cinco ejemplares de A. azarae (trivirgatus), por los que creyeron que se trataba de un grupo familiar, pero al abandonar, estos individuos se reagruparon en grupos de dos y tres individuos respectivamente.

A sumimos que la tolerancia por el uso de frutos en un mismo árbol ocurre cuando existen ramas grandes y esparcidas, copa frondosa y abundante, 
frutos que facilitan la concurrencia simultánea de dos o más grupos, sin la ocurrencia de encuentros hostiles.

\section{Competición con otros mamíferos nocturnos}

Aotus, a pesar de sus hábitos nocturnos, ocasionalmente tiene como competidores por los recursos alimenticios a primates diurnos como Cebus y Saimiri. Estos han demostrado cierta actividad nocturna, principalmente en noches de luna llena, llegando incluso a desplazar a los Aotus del árbol frutal (Wright 1985). A simismo, existen otros mamíferos de hábitos similares a los Aotus, que además de consumir los mismos recursos alimenticios, también ocupan los mismos estratos del bosque y utilizan los mismos ambientes constituidos por huecos y concavidades para el descanso o "sueño diurno".

Como competidores de Aotus por los frutos hemos registrado a Potos flavus y a quirópteros (Tabla 2). Estas competiciones ocurrieron con mayor frecuencia en árboles de Eschweilera sp. (40\%) y Ficus sp. (30\%). De estos dos mamíferos $P$. flavus es el principal competidor por su tamaño y peso superior a Ios demás competidores (2.0 kg como promedio, Terborgh 1985), y por ser catalogado básicamente como frugívoro (Walker et al. 1964).

Como resultado de las competiciones hemos observado casos de hostigamiento por la posesión del arbol alimenticio entre A otus y $P$. flavus hasta en dos oportunidades. El primero ocurrió en las inmediaciones de la localidad de $\mathrm{M}$ ishana, en un árbol de Ficus sp., de aproximadamente $25 \mathrm{~m}$ de alto, donde estuvieron simultáneamente un grupo de $A$. vociferans con tres individuos y dos ejemplares adultos de $P$. flavus, separados por las ramas frondosas y esparcidas en un radio de $20 \mathrm{~m}$ de copa. Inicialmente el grupo de A otus se encontraba en el árbol, luego ingresaron al árbol dos ejemplares de $P$. flavus;, por espacio de 32 minutos ambas especies se alimentaron de los frutos en ramas diferentes, hasta que Potos se trasladó a una rama cercana a los Aotus, reaccionando éstos con vocalizaciones guturales de series combinadas, cortas, fuertes y aceleradas interrumpidamente pero con menor intensidad al finalizar, lo que conlleva a un tipo de comportamiento hostil descrito por Moynihan (1964). Por su parte, P. flavus emitió vocalizaciones fuertes, intermitentes y prolongadas, en un acto probable de agresión al competidor eventual. Como resultado del intercambio de vocalizaciones, Aotus optó por la retirada, mientras que Potos continuó alimentándose en el árbol.

Un segundo caso de competición entre $P$. flavus y Aotus fue observado en un árbol de Ficus sp. En la cuenca del río Napo. En esta oportunidad el competidor toleró la presencia de Aotus y cada cual abandonó el árbol al amanecer. Estas observaciones corroboran las observaciones de W right (1985), quien además manifiesta que Potos, Didelphis y Bassaricyon son las únicas 
especies nocturnas que por su tamaño podrían desplazar a los Aotus del árbol frutal.

En cuanto a los quirópteros, en circunstancias distintas a las anteriores escuchamos y observamos en más de una oportunidad murciélagos volando alrededor de los árboles donde se alimentaban tanto Aotus como Potos. La competición fue confirmada cuando al examinar restos de frutos caídos al piso del bosque, éstos presentaban huellas de dientes puntiagudos. Los frutos en algunos casos presentaron desgarramiento del mesocarpio en pequeñas rodajas y en otras finísimas hendiduras del pericarpio. Estas observaciones coinciden con Estrada et al. (1984), quienes afirman que los murciélagos no eluden la presencia de primates u otros mamíferos cuando están alimentándose en el mismo árbol.

Finalmente, hemos observado en repetidas oportunidades la concurrencia de Bassaricyon gabbii y caluromys lanatus al mismo árbol donde Aotus se alimentaba, pero en diferentes horas. Esta conducta indicaría que estas especies en lo posible tratan de evitar acciones competitivas con hostigamiento.

\section{CONCLUSIONES}

4.1 Frutos de Ficus sp. e Inga marginata, así como flores y néctar de Eschweilera sp. y Ficus sp. Fueron componentes más frecuentes en la dieta alimenticia de $A$. vociferans.

4.2 Los frutos consumidos por A. vocifernas en su mayoría se caracterizan por su sabor dulce y agradable, siendo el mesocarpio la parte más utilizada.

4.3 A. vocjferans también tiene como competidores por el recurso alimenticio a Potos flavus y a los quirópteros frugívoros.

4.4 La competición alimenticia intraespecífica y con otros mamíferos ocurre cuando hay sobreposición parcial de territorio y coincidente con la temporada de escasez de frutos.

\section{AGRADECIMIENTOS}

Expresamos nuestra gratitud a Gilberto Asipali, Amanojo Isuiza, DesiderioMarichi y Milton Ahuanari, quienes participaron como guías y asistentes de campo.

A simismo, hacemos extensivo nuestro agradecimiento al Dr. Juan E. García (Estación Biológica de Doñana, Espafía) y al Dr. James Penn (A mazon 
Conservation Fund, USA) por el aporte crítico al manuscrito. Este trabajo fue financiado por el Proyecto peruano de Primatología "M anuel Moro Sommo" (Carta Convenio ICF/ZNS/O10, entre el gobierno del Perú y la Organización Panamericana de la Salud).

\section{BIBLIOGRAFIA}

A QUINO, R. 1986. Characteristics and use of sleeping sites in A otus (Cebidae: Primates) in the amazonian lowland of Perú. Am. J. Primatol. 11:310331.

AQUINO, R. y F. ENCARNACION, 1986. Population structure of A otus nancymai (Cebidae: Primates) in Peruvian amazon lowland forest. A m. J. Primatol. 1:1-7.

ENCARNACION, F., 1985. Introducción a la flora y vegetación de la amazonía peruana: Terminología y breve descripción de las formaciones vegetales de la llanura amazónica. Candollea. 40:237-252.

ESTRADA, A., C. COATES-ESTRADA, VASQUES-YAÑEZ y A. OROSCO SEGOVIA, 1984. Comparison of frugivory by honling monkeys (Alouatta palliata), and bats (Artibeus jamiacensis) in the tropical ram forest of Las Tuxtlas, M exico. A m. J. Primatol. 7:3-13.

GARCIA, J. F. BRAZA, 1987. A ctivity rhythms and use of a group of Aotus azarae in Bolivia during the raining season. Primates. 28(3):337-342.

HERNANDEZ-CAMACHO, J. y R. COOPER 1976. The nonhuman primates of Colombia. Pp. 35-69 en Thorington, R. y Jr. P. Hetne (ed.) N eotropical Primates. Nat. A cad. Sci. Washington D.C.

HERSHKOVITZ, P., 1983. Two new species of night monkeys genus Aotus (Cebidae: Platyrrhini): a preliminary report on A otus taxonomy. A m. J,. Priamtol. 4:209-243.

JANSON, C. y C. VAN SCHJL, 1988. Recognizing the many faces of primate food competition: M ethods. Behavior. 105:165-186.

MOYNIHAM, M., 1964. Some behavior patterns of Platyrrhine monkeys. Smithsoninan M isc. Collections. 146(5): 1-84. 
RATHBUN, G. y M. GACHE 1977. The status of Aotus trivirgatus in A rgentina. Centro A rgentino de Primates y $\mathrm{N}$ ational Institutes of $\mathrm{H}$ ealt Report, Washington D.C.

----1980. Ecologycal survey of the night monkeys, Aotus trivirgatus, in Formosa Provinee, A rgentino. Primates. 21:211-219/

THORINGTON, R., N. MUCKENHJRN Y G. MONTGOMERY, 1976. Movements of a wild night monkeys, Aotus trivirgatus. Pp. 32-34 en Thorington, R. y Jr. P. Heltne (ed.). Neotropical Primates. Nat. A cad. of Sci. Washington D.C.

TERBORGH, J. 1985. Frugívoros en la comunidad de vertebrados de Cocha Cashú Reporte Manú. Centro de datos pra la conservación, UNA. Lima, Perú.

------y CH. JANSON, 1983. Ecology in Southeasterns Peru. National Geographic Society Research Reports. 15:655-662.

WALKER, E. E. WARNICK, K. LANGE, H. UIDIBLE, 5. HAMLET, M. DAVIS, Y P. WRIGHT, 1964. Mammals of the world, ira. edición, Vol. 1. John Hopkins Press, Baltimore, USA.

WRIGHT, P. , 1978. Home range activity pattern and agonistic encounters of a group of night monkeys (Aotus trivirgatus) in Perú. Folia Primatol. 29:43-55.

1981. The night monkeys; Genus A otus. Pp. 211-240 en Coimbra-Filho y R.M ittermeir (ed.). Ecology and Behavior of Neotropical Primates. A cademia Brasileira de Ciencias, Rio de Janeiro.

1985. the cost, and benefits of nocturnality for Aotus trivirgatus. In tbe night monkey. University International, A nn A rbor, MI, USA. 
FIGURA 1: Ubicación geográfica del área de estudio

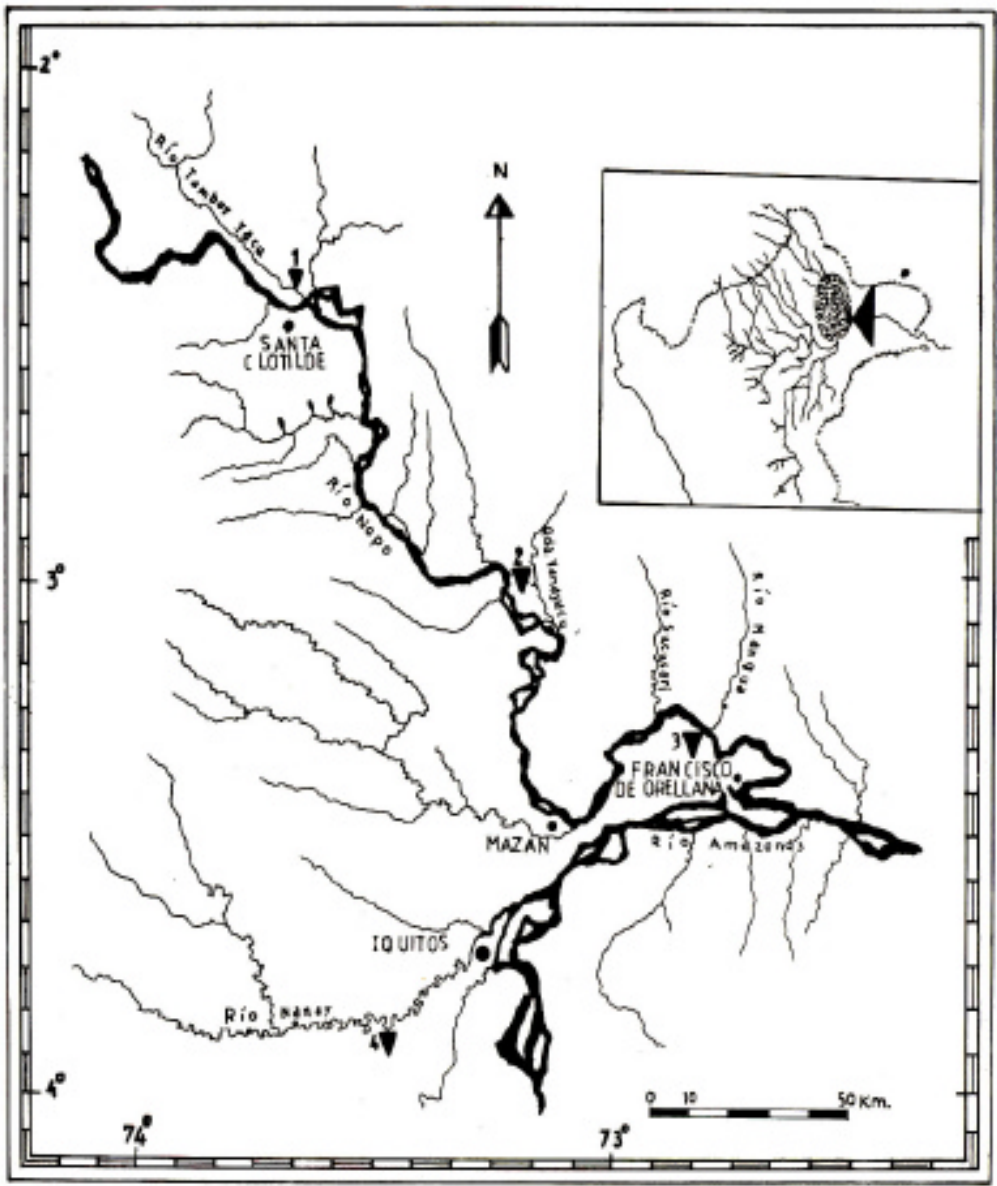


TABLA 1. Registro de plantas consumidas por Aotus vociferans en la cuenca de los ríos Napo y Nanay.

\begin{tabular}{|c|c|c|c|c|c|c|}
\hline FAMILIA & ESPECIE & NOMBRE LOCAL & Frutos & $\begin{array}{c}\text { A L I M } \\
\text { Flores y } \\
\text { néctar }\end{array}$ & $\begin{array}{l}\text { E N T } \\
\text { Total }\end{array}$ & \% \\
\hline Apocynaceae & Parahancornia peruviana & Naranjo podrido & 1 & - & 1 & 3.45 \\
\hline Arecaceae & Iriartea delboidea & huacrapona & 2 & - & 2 & 6.90 \\
\hline Burseraceae & Protian sp. & copal huayo & 1 & - & 1 & 3,45 \\
\hline Clussiaceae & Rheedia gardneriana & charichuclo & 2 & - & 2 & 6.90 \\
\hline Lecythidaceae & Eschweilera sp. & machimango & - & 3 & 3 & 10.33 \\
\hline Leguminosae & Inga edulis & guaba & 1 & - & 1 & 3.45 \\
\hline Leguminosac & Inga marginata & shimbillo & 3 & - & 3 & 10.33 \\
\hline Leguminosae & Inga sp. & shimbillo & 2 & - & 2 & 6.90 \\
\hline Melastomataceac & Bellucia axinanthera & nispero & 1 & - & 1 & 3.45 \\
\hline Moraceae & Ficus sp. & renaco & 4 & 2 & 6 & 20.69 \\
\hline Momceae & Pourouma minor & sachauvilla & 2 & - & 2 & 6.90 \\
\hline Moraceae & Cecropia sp. & cetico & 1 & - & 1 & 3.45 \\
\hline Polygonaceac & Coccoloba sp. & vino huayo & 1 & - & 1 & 3.45 \\
\hline Rubiacene & Rudgea retifolia & pichico runto & 1 & - & 1 & 3.45 \\
\hline Myrtaceae & Eugenia sp. & rupiña & 1 & - & 1 & 3.45 \\
\hline Sapotaceae & Pouteria sp. & caimitillo & 1 & - & 1 & 3.45 \\
\hline TOTAL & & & 24 & 5 & 29 & 100.00 \\
\hline
\end{tabular}

TABLA 2. Registro de otros mamiferos nocturnos competidores de Aotus vociferans

\begin{tabular}{|c|c|c|c|c|}
\hline \multirow{2}{*}{$\begin{array}{l}\text { Mamífero } \\
\text { competidor }\end{array}$} & \multicolumn{2}{|c|}{ Plantas usadas } & \multirow[b]{2}{*}{$\begin{array}{c}\text { Flores y } \\
\text { nectar }\end{array}$} & \multirow[t]{2}{*}{$\%$} \\
\hline & Especie & Fruto & & \\
\hline $\begin{array}{l}\text { Bassaricyon gabiii } \\
\text { (olingo) }\end{array}$ & Inga edulis & 1 & - & 10 \\
\hline $\begin{array}{l}\text { Caluromys lanatus } \\
\text { (Chosna pericote) }\end{array}$ & Pourouma minor & 1 & - & 10 \\
\hline Potos flavus & Inga marginata & 1 & - & \multirow{4}{*}{50} \\
\hline \multirow[t]{2}{*}{ (Chosna) } & Ficus sp. & 2 & - & \\
\hline & Eschweilera sp. & - & 2 & \\
\hline Quirópteros & Eschweilera sp. & - & 2 & \\
\hline frugívoros & Ficus sp. & 1 & $=$ & 30 \\
\hline TOTAL & & & & 100 \\
\hline
\end{tabular}

